\title{
Comparison of 2D and 3D ultrasonic pulse echo imaging techniques for structural assessment.
}

\author{
Lubos Misak $^{1}$ and David Corbett ${ }^{2}$, Michael Grantham ${ }^{3}$ \\ ${ }^{1}$ Proceq SA, Proceq Europe Department, 8603 Schwerzenbach,Swizerland \\ ${ }^{2}$ Proceq SA, Product Development Department, 8603 Schwerzenbach,Swizerland \\ ${ }^{3}$ Leeds University and Sandberg LLP, London, UK
}

\begin{abstract}
The use of ultrasonic waves in the medical field is quite advanced, especially in the area of three-dimensional (3D) imaging of the human body and is quite common. Also, in Non-Destructive Testing of metal (for example weld checking), flaw detection with reliable 3D imaging is already in use. In the past $1-2$ years, the construction industry has made significant advances in two-dimensional (2D) and three-dimensional (3D) imaging of concrete structures, for finding problems, or irregularities inside, or just for confirming the quality of the construction work. This paper describes the principles of the different types of scan, A Scan, B Scan etc and how the scans can be combined to make 3D images. The advantages of multi head array systems in providing more detail and revealing depth information are shown. This paper will show the advantages of this development and how it can ease workflow.
\end{abstract}

\section{Introduction}

The use of ultrasonic waves in the medical field is quite advanced, especially in the area of three-dimensional (3D) imaging of the human body and is quite common. Also in Non-Destructive Testing of metal (for example weld checking), flaw detection with reliable 3D imaging is already in use.

In the past $1-2$ years, the construction industry has made a big step in two-dimensional (2D) and threedimensional (3D) imaging of concrete structures, for finding problems, or irregularities inside, or just for confirming the quality of the construction work.

This has been made possible by progress in the electronics industry (smaller, faster microchips, high resolution of screens) and the possibility to handle a big volume of data, with powerful software algorithms to produce and display two-dimensional (2D) and threedimensional (3D) objects.

This paper will show the advantages of this development and how it can ease workflow.

\section{Recent Research}

Khairi et al (1) discussed the theory behind ultrasonic tomography and illustrated it with a number of applications cross-industry, but including its application in concrete and mortar and in evaluation of damage to structures.

Scott (2) discussed the use of ultrasound computed shear wave tomography for the inspection of Nuclear Structures to detect hidden defects.
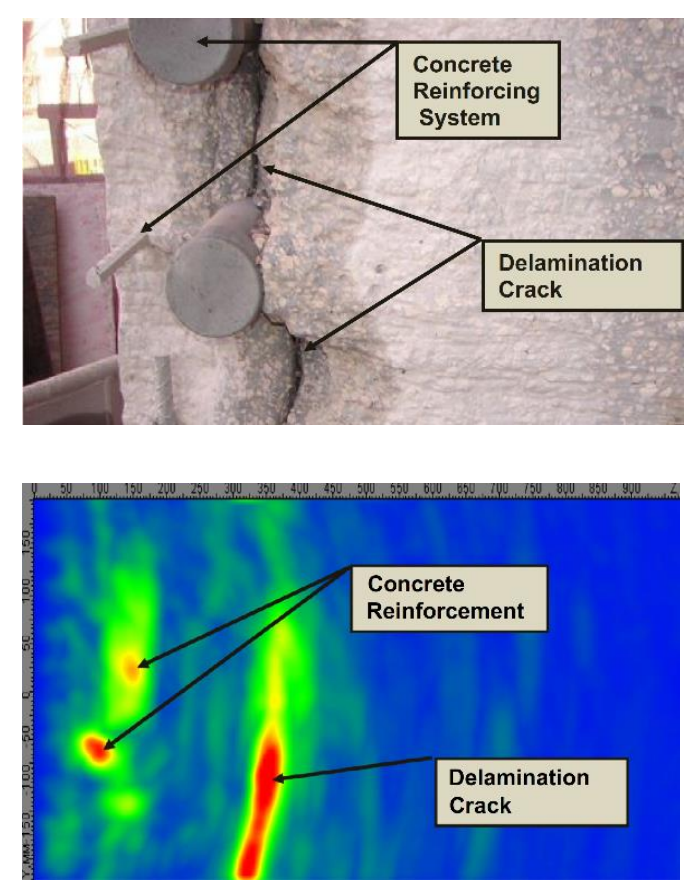

Fig 1. Delamination in a nuclear containment structure wall (top) and an ultrasound tomographic scan (bottom) - after Scott (2).

Schabowicz, (3) discussed the principle of the technique, illustrating its use on laboratory test specimens and also on a cracked, defective foundation slab, suspected of being too thin and being found by tomography to be noncompliant with its design. 
Choi et el (4) described a comprehensive visualization scheme to characterize reinforced concrete structures, based on integrated ultrasonic tomography and 3-D computer vision. Their paper describes a recently developed hybrid aircoupled/contact ultrasonic transducer system which enables the generation of internal tomographic images that characterize the interior condition of structures. for example the presence of voiding defects, Based on tomographic cross-sectional slice images, 3-D volumetric internal images were built up to obtain full stereoscopic analysis of full-scale reinforced concrete elements.

Haach and Ramirez (5) described the application of ultrasonic tomography for the qualitative assessment of concrete. They conducted several experimental tests with variations in concrete composition, presence of voids, arrangement and frequency of transducers on concrete prisms. The results showed that ultrasonic tomography was more efficient than conventional ultrasonic tests. Analyses of several transducer arrangements revealed a large number of measurements did not necessarily return better images.

\section{A-scan principles}

The classical measurement with Ultrasonic transducers (sender - receiver) looks like this:

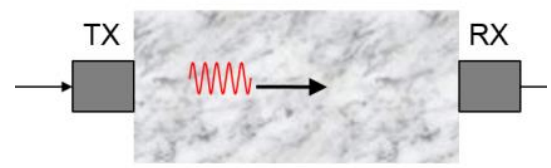

Fig 2 Normal UPV direct transmission

An ultrasonic signal is from transmitter transducer to the receiver transducer through the material and results in an A-scan.

The A-scan presentation is a display of the ultrasonic signal in which the $\mathrm{x}$-axis represents the time and the $\mathrm{y}$ axis the amplitude

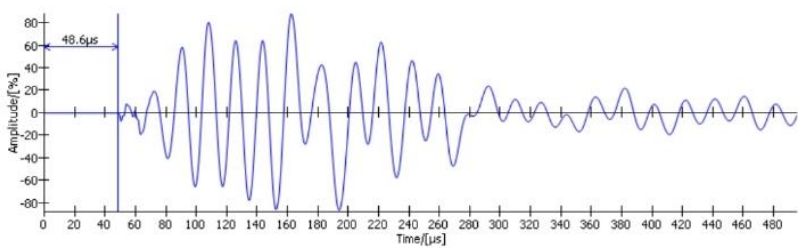

Fig 3. Typical UPV Trace showing signal amplitude vs time of flight. The $48.6 \mu$ s represents the time for the first receipt of signal.

This procedure requires the use of a couplant to ensure a good contact between the transducer and the material (concrete, brick, mortal, ..) and the measurement is usually taken directly, semi-directly, or indirect (on the surface).

\section{B-scan principles}

The B-scan presentation is an image of the results of an ultrasonic examination showing a cross section of the test object perpendicular to the scanning surface and parallel to the reference direction. The cross section will normally be the plane through which the individual A-scans have been collected.

The B-scan is principally a series of A-scans in a line in one direction. For example, in one axis (X-axis) this would be a B-scan.
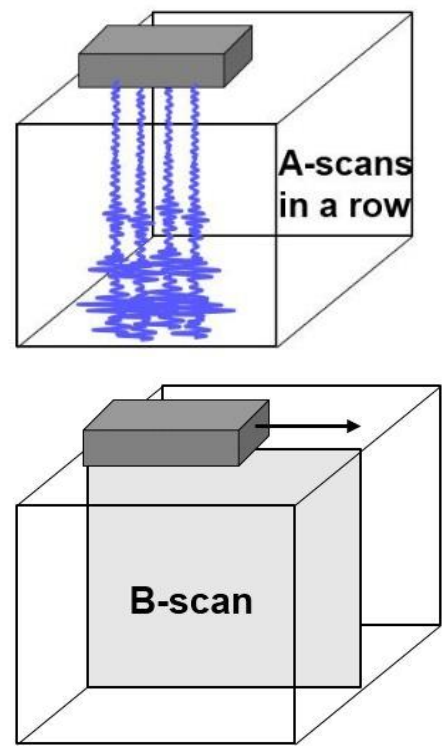

Fig. 4 The "B" Scan principle

\section{C-scan, D-scan, P-scan, Volume scan}

The same scan in the perpendicular axis is often referred to as a D-scan.

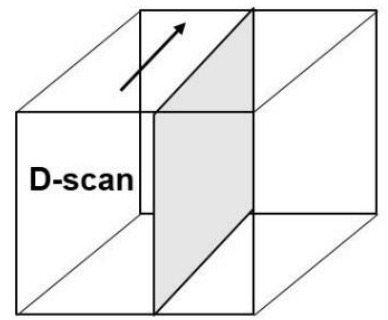

Fig 5 The "D" Scan Principle

The C-scan presentation is an image of the results of an ultrasonic examination showing a cross section of the test object parallel to the scanning surface. The evaluated parameter in the image is the amplitude of the signal.

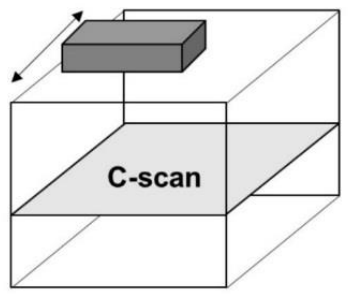

Fig 6 Typical "C" Scan 
P-scan (time slice) and Volume scan. A projection view of several B-Scans or C-Scans. Once we have a volume scan we can look at gated slices in each of the three dimensions, at any depth we choose.
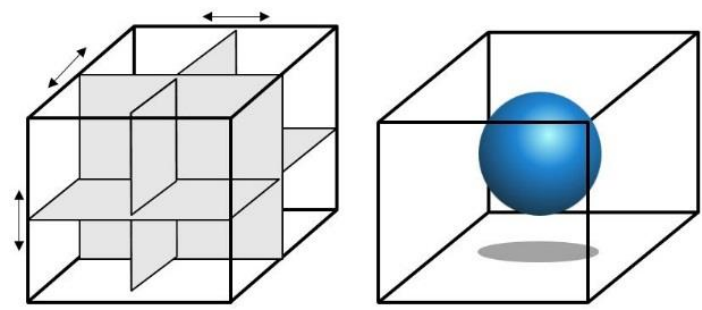

Fig 7. "P" Scan (Left) and Volume Scan (Right)

\section{DPC transducers and Array Probe.}

The development of DPC (Dry Point Contact) transducers have been a significant advance and have helped to have contact with the material without using couplant.

These transducers are used in pulse echo mode in which the transmitter and receiver are on the same side which is an advantage, if there is access only from one side (tunnels, subways, bridges, ...). This technique allows reflections from internal flaws to be obtained (object detection inside), or to detect the thickness of the object from the back-wall echo.
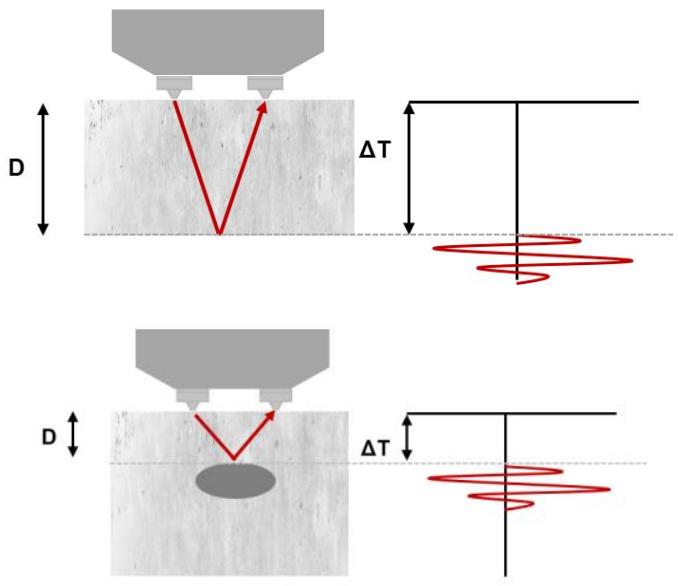

Fig 8. Typical "one side" UPV scan where reflections are used to see defects or the back wall in a structure.

The technique is further improved by using multiple channels in an array transducer, enabling faster scanning of bigger areas.

The working principles are, that one channel transmits and the others are listening, each receiving a separate Ascan, then the second channel transmits and the others are listening and so on. This continues in a cycle and the resulting A-scans are used to create a B-scan in real time.

The principle is shown in Figure 8 where the clear advantages in time and resolution using a multi-scanning system are obvious.

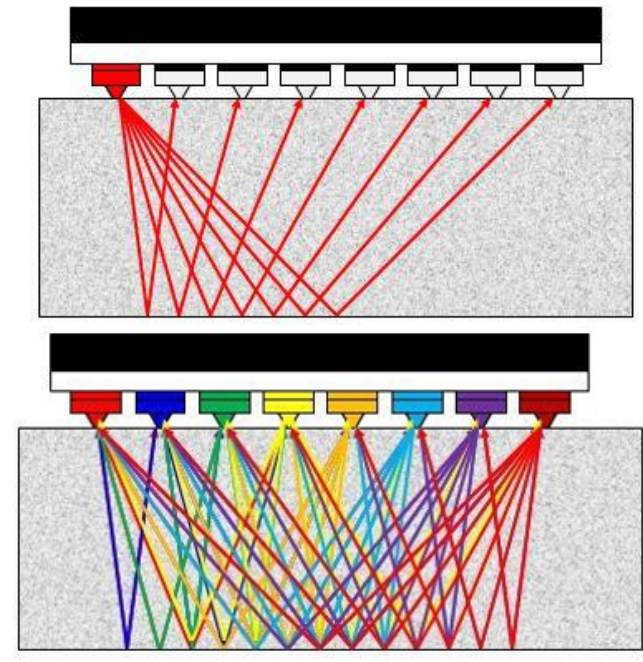

Fig 9. Multi scanning array as used in advanced ultrasonic tomography.

It is also possible to combine array transducers together to double the scanning surface. This reduces the scanning time.

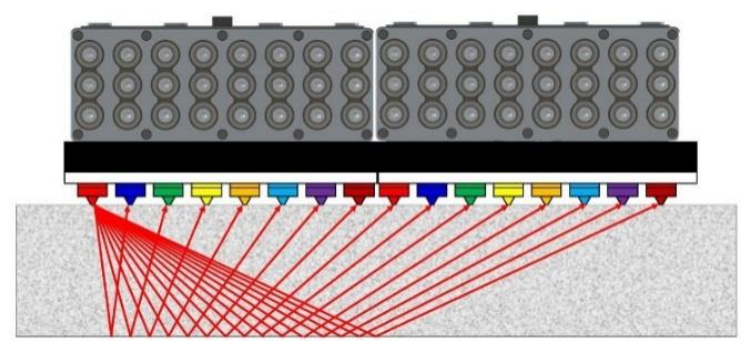

Fig 10. Combining two transducer arrays to speed up scanning.

\section{Comparison of 2D and 3D scans.}

Having access to both $2 \mathrm{D}$ and $3 \mathrm{D}$ scans provides the user with more information about the test object. The 2D images are in the $\mathrm{X}$ and $\mathrm{Y}$ axis (B-scans), and provide depth information about objects. Combination and interpolation of $\mathrm{B}$-scans allows $3 \mathrm{D}$ images to be created which then allow the user to look at the amplitude information of the entire scan area at a chosen depth. This provides information about the extent of objects or defects.

In Fig 11a we can see two objects, very clearly. To determine the extent of such objects it is possible to use the $3 \mathrm{D}$ scan set to the depth of the object in question and in the following images $-11 \mathrm{~b}, 11 \mathrm{c}$ and $11 \mathrm{~d}$ which show information at different depths, it is clear to see that it extends throughout the length of the scan and is almost certainly a pipe of sorts. (In fact, the object in this case was a tendon duct.) Accurate depth information is determined by detailed analysis of the A-scan at any particular location. 


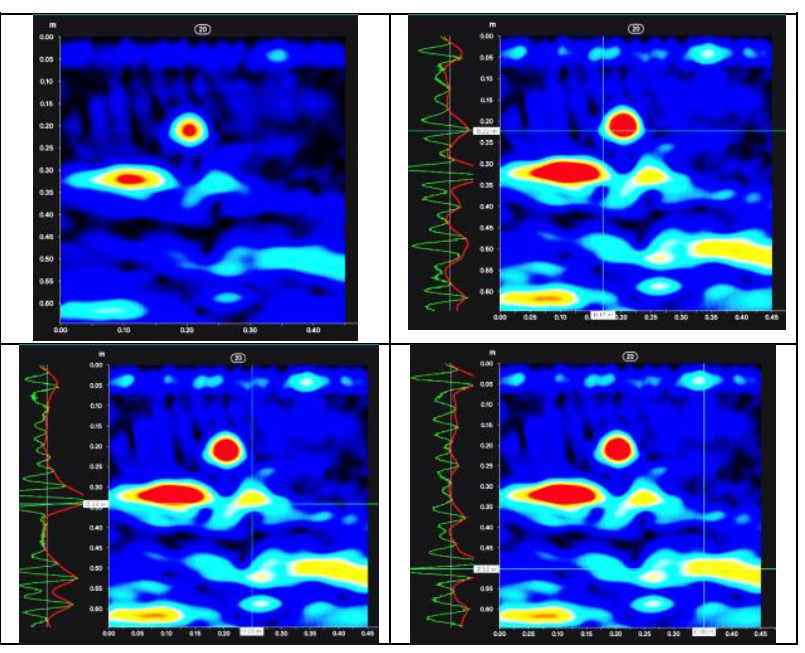

Fig 11 clockwise from top left: a,b,c,d

With the 3D scan, we have a volumetric model of scanned structure inside of the object, which we can see and rotate to get a visual appreciation of the spatial relationship between objects detected, even quite complex ones as in fig 12 .
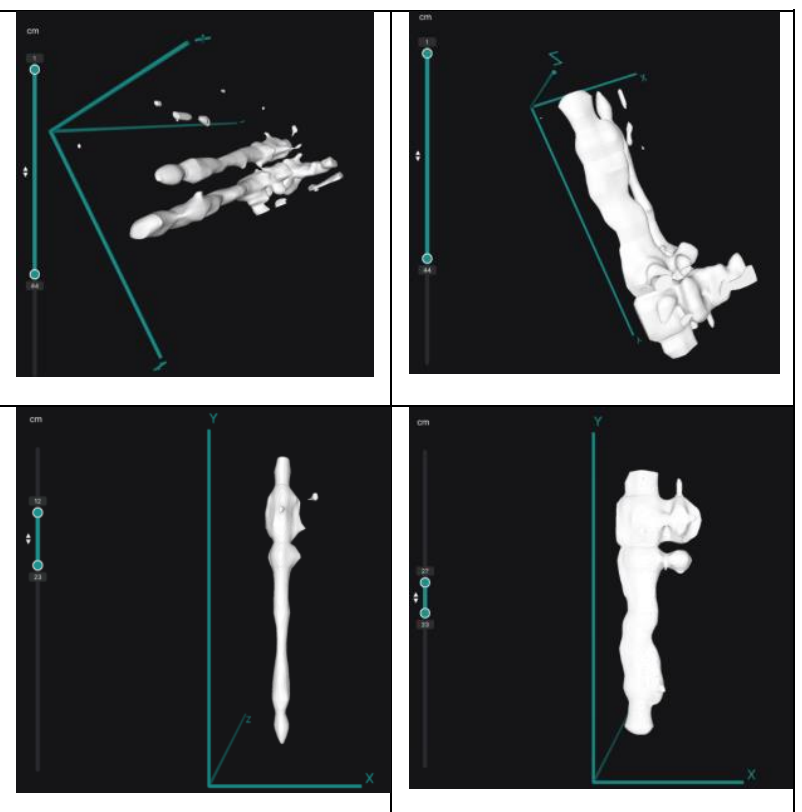

Fig 12. Complex object visualized and rotatable in $3 \mathrm{D}$

Also, in this type of representation it is important to be able to blend out sections of the scan or increase the gain at various depths. For example, rebars are not so easily detected by ultrasonic pulse echo instruments, but they can be made visible by increasing the gain and lowering the pulse velocity. However, these actions have the effect of distorting the location of deeper objects.

\section{Conclusions}

Ultrasonic pulse echo scanning has developed significantly in recent years, but the main issue remains in interpretation of the images. Both 2D and 3D images are useful to the engineer for assessing a structure. Each provides specific information that is essential in trying to make sense of the scanned structure. The advanced hardware and software technique for ultrasonic testing is making it possible to create better images of the object for technicians and engineers, but also make simple to understand $3 \mathrm{D}$ renders which are much easier to explain to non-NDT specialists. The latest advances are even making use of techniques such as Augmented Reality (AR) which allow the scan to be viewed in-situ on a tablet and even to be able to draw in the position of bars etc. on the structure surface using a special marker which is visible on the tablet and can then mark the positions of bars from the virtual image.

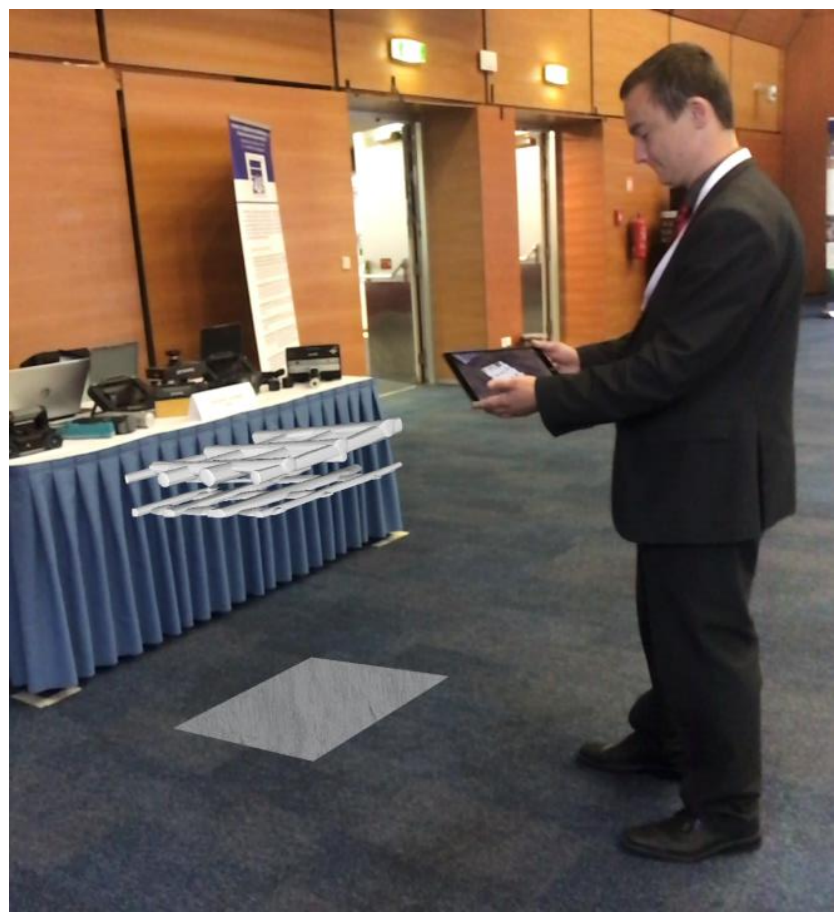

Fig 13. Graphic depicting the future of internal visualization of structures.

The 2D pictures are more suitable for detail analysis, for specialists, where they can put more energy into the picture, check the A-scan and go for details but for non specialists the power of visualizing and exploring inside a structure in an understandable way is a huge advance. Undoubtedly, the technique will continue to advance and better, improved techniques and visualisations will become available.

\section{References}

1. M. T. M. Khairi, S. Ibrahim, M. A. M. Yunus, M. Faramarzi, G. P. Sean, J. Pusppanathan, A. Abid, Measurement, 146, 490-523, (2019).

2. D.B. Scott, Energy Conversion and Management. 74, 582-586, (2019).

3. K. Schabowicz, Archives of Civil and Mechanical Engineering, 14, 295-303, (2014).

4. H. Choi a, Y. Hamb, J S. Popovics, Construction and Building Materials 123, 384-393 (2016) 
5. V.G. Haach, F. C. Ramirez, Construction and Building Materials 119 61-70 (2016)

6. D. Corbett, One Belt One Road Concrete Technology Forum, Singapore (2018)

7. D. Corbett, Pundit 250 Array Application (2019) 